\title{
Performance Analysis of Channel Capacities in Different Diversity Techniques
}

\author{
${ }^{1}$ Samit Kumar Ghosh, ${ }^{2}$ Jishan Mehedi \\ Dept of ECE, MLR Institute of Technology, Hyderabad, Telangana, India, Dept of ECE, Jalpaiguri Government \\ Engineering College, Jalpaiguri, West Bengal, India \\ Email:samitnitrkl@gmail.com, j.mehedi@gmail.com
}

\section{Received: 20 ${ }^{\text {th }}$ December 2017, Accepted: 20th January 2018, Published: 28th February 2018}

\begin{abstract}
In broadcast communications, a diversity scheme refers to a technique for enhancing the reliability of a message signal by utilizing at least two correspondence channels with various attributes. In this paper we consider all four possible scenarios: Multiple-input and multiple output (MIMO), multiple-input and single-output (MISO), single input and multi-output (SIMO), and single-input and single-output (SISO) systems. We derive the closed form expressions of channel capacity, and reformulate them irrelevant to the channel of the eavesdropper. This makes our scheme valid when the transmitter does not know any information about the eavesdropper. We investigate the solution for the capacity problem in SISO scenario at first, which can be extended to SIMO, MISO, and MIMO systems. We also analyze the channel capacity of all four types of scenarios. The comparative studies show that the channel capacity of MIMO system is higher.
\end{abstract}

Keyword: Diverity, Channel Capacity,SISO, MIMOI

\section{Introduction}

Future generation of cellular communication require higher data rates and a more reliable transmission link. The information rates can be enhanced by expanding the transmission data transmission and utilizing higher transmission control. Wireless communication channels suffer from various factors such as fading problems. A diversity technique is used to improve the performance of these fading channels. In diversity techniques communication channels are supplied with multiple transmitting and receiving antennas. The signal is transmitted and received through different ways. As a result, the probabilities that all replicas of signals will fade simultaneously is reduced considerably. Concerns on wireless communication security grow rapidly with the wide application and popularity of wireless communication because it is easy to overhear communication over wireless medium. An eavesdropper can receive the radio signal from a transmitter to its intended receiver for the broadcast nature of wireless medium. Consequently, eavesdroppers can acquire the channel state information (CSI) between the transmitter and the receiver. On the contrary, the transmitter usually knows nothing about the eavesdropper, such as its location, its CSI, even its presence. Physical layer security technology aims to change the adverse situation and provide secure communication in physical layer. In the study of physical layer secrecy, it is assumed that the transmitter knows nothing about the eavesdropper, but the eavesdropper has acquired what are needed for the communication. This situation makes more difficult to realize secure communication.

\section{SYSTEM BASED ON SPACE DIVERSITY}

Diversity is one of the dominant communication techniques that provide wireless link improvements at comparatively low cost. At the receiver end it provides two or more inputs such that the fading phenomena among these inputs are uncorrelated. Space diversity is one of the wireless diversity schemes in which two or more antennas are used for which the quality and reliability of a wireless link is improved. The quality and effectiveness of any wireless link is depends upon three basic link performance parameter.

- $\quad$ Speed (or spectrum)

- Range (or coverage) and

- Reliability (or security).

The utilization of different waveforms transmission in parallel constitutes another kind of radio communication which is utilizing multi-dimensional signals which is the best approach to enhance every one of the three essential connection execution parameters utilizing various receiving wire framework. The measurement of the capacity of a wireless link is done by bits per second per Hertz (bps/Hz) [8-10].

Wireless systems are classified into four types which are important to the study of space diversity. The space diversity again has two categories:

- Macro diversity: In the event that the receiving antennas are at a far separation, for instance, at various cell base stations sites or WLAN access point.

- Micro diversity: In the event that the receiving are at a distance in the order of one wavelength.

Presently, four types of systems can be categorized as far as diversity is concerned (input and output refers to the number of antennas). 
A. Single Input Single Output- The SISO system is typically less complex and deals with communication between a transmitter and a receiver. In this communication systems two antennas is used one for source (Transmitter) and another for destination (receiver) which is depicted in figure 1. In SISO, error probability is critically damaged by fading and it can cause a reduction in data speed. Smart antenna technology such as SIMO, MISO, and MIMO is used in order to minimize or eliminate a problem which is caused by multipath propagation.
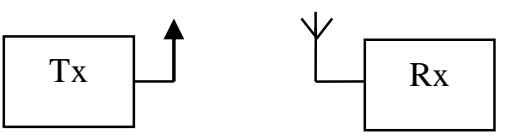

Fig 1: SISO Communication Systems

B. Single Input Multi Output- In this communication systems one antenna is used for source (Transmitter) and two or more antennas are used for destination (receiver) which is depicted in figure 2 . It is also referred to as receive diversity. It is frequently used to empower a recipient framework that gets signals from various autonomous sources to combat the effect of fading.
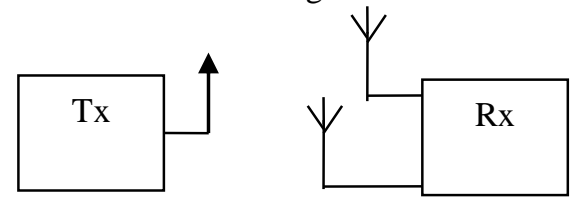

Fig 2: SIMO Communication Systems

C. Multi Input Single Output- It is also referred to as transmit diversity. For this situation, similar information is transmitted needlessly from the two transmitter receiving wires. The recipient is then ready to get the optimal signal which it would then be able to use to get extricate the required information. The benefit of utilizing MISO is that the various radio wires and the repetition coding/handling is moved from the receiver to the transmitter. In occurrences, for example, mobile phone UEs, this can be a noteworthy favorable position as far as space for the reception apparatuses and lessening the level of preparing required in the receiver for the excess coding. This positively affects size, cost and battery life as the lower level of preparing requires less battery utilization.
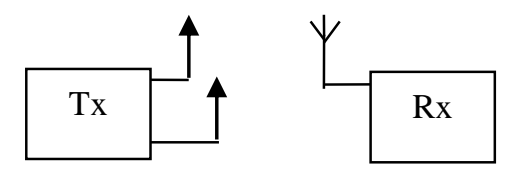

Fig 3: MISO Communication Systems

D. Multi Input Multi Output- Multiple Input, Multiple Output (MIMO) innovation offers the potential for a significant increment in capacity and execution inside a given data transmission and power spending plan. MIMO is adequately a radio reception apparatus innovation as it utilizes various receiving wires at the transmitter and receiver to empower an assortment of flag ways to convey the information, picking separate ways for every radio wire to empower multiple signal paths to be utilized.

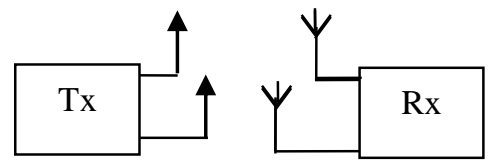

Fig 4: MIMO Communication Systems

ChANNel CAPACITIES OF SISO, SIMO, MISO, MiMO In this section, the expression for evaluation of channel capacity is described. According to Shannon-Hartley theorem, the channel capacity is specified by

$$
C=B \log _{2}(1+S N R)
$$

Where, $\mathrm{C}$ is capacity, $\mathrm{B}$ is bandwidth, SNR is signal to noise ratio. From the above equation it can be known that, an increase in a channel's SNR results in marginal gains in channel throughput. So for achieve the higher data rates in communication channel is by increasing the bandwidth of the signal. For wide data transmission channels, one incomplete answer for understanding the multipath challenge is to utilize a progression of narrowband covering subcarriers.

For SIMO system, we have M number of receiving antennas at the receiver end. If the received signal amplitude on these antennas are same then they can be coherently added together to form the increase signal power $\mathrm{M}^{2}$ times [11-12]. Hence, the increase SNR is equivalent to

$$
\begin{array}{r}
S N R^{\prime}=\frac{(M \times M) .(\text { Signal Power })}{M .} \\
=M \frac{\text { Noise Pwer }}{\text { Noignal Power Power }}
\end{array}
$$

So the channel capacity becomes

$$
C=B \log _{2}(1+M . S N R)
$$

In the case of MISO system we have $\mathrm{N}$ number of transmitting antenna for which the total power which is transmitted is divided into $\mathrm{N}$ branches. In this communication system only one receiving antenna is exists so the noise level is same as consider to the SISO. Thus the overall SNR is increased and it is approximately

$$
\begin{array}{r}
S N R^{\prime}=\frac{(N \times N) \cdot \frac{\text { Signal Power }}{N}}{\text { Noise Power }} \\
=N \frac{\text { Signal Power }}{\text { Noise Power }}
\end{array}
$$

So the channel capacity for this case $C=$ $B \log _{2}(1+N . S N R)$ 
MIMO is a combination of MISO and SIMO systems. In this system the overall SNR is increased into $M N$ times and it is approximately

$$
\begin{array}{r}
S N R^{\prime}=\frac{(M \times M)}{(N \times N) \cdot \frac{\text { Signal Power }}{N}} \\
\text { M. Noise Power } \\
=\text { M.N } \frac{\text { Signal Power }}{\text { Noise Power }}
\end{array}
$$

So the channel capacity becomes $C=B \log _{2}(1+$ M.N.SNR)

\section{Simulation Results}

The performance of capacity comparison of SISO, SIMO, MISO and MIMO system depicted in figure 5 and the analysis results and comparative analysis is shown in Table 1 and Table 2 respectively. Here $\mathrm{N}$ represents transmitting antenna and $\mathrm{M}$ represents receiving antenna of communication systems.

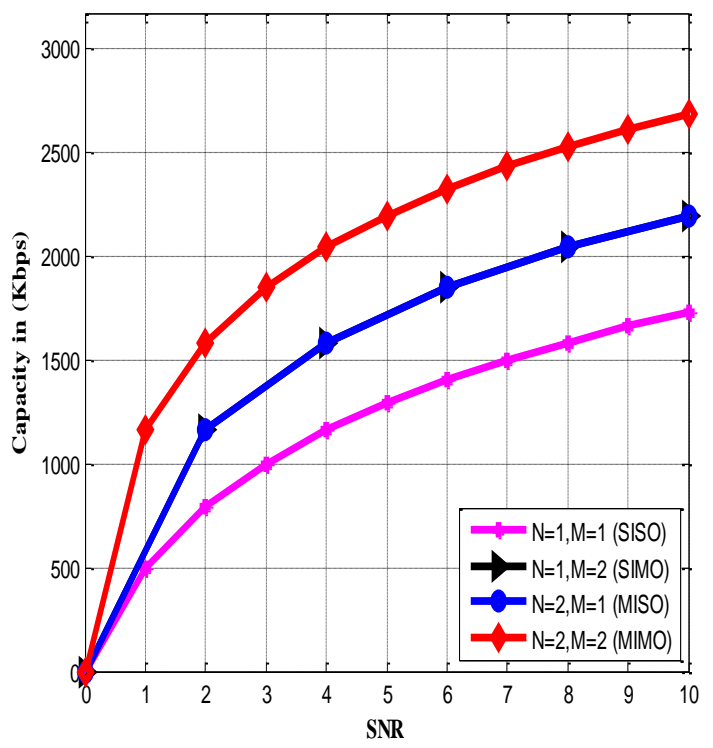

Fig 5: Performance Capacity Comparison

By analyzing from the simulation, it can be concluded that for the SISO system the channel capacity is lesser than compare to SIMO, MISO and MIMO system. In SISO system, only one single path in between transmitter and receiver and single antenna is in transmitter and also receiver. Its channel capacity is poor yet framework configuration is particularly less demanding. In simulation, SNR versus capacity companion is same in the scenarios of SISO/SIMO and in case of MIMO it is higher.

Table 1: Result Analysis

\begin{tabular}{|c|c|c|c|c|c|}
\hline Sl & SN & \multicolumn{4}{|c|}{ Channel Capacity (Kbps) (Simulated) } \\
\cline { 3 - 6 } No & $\mathrm{R}$ & $\begin{array}{c}\mathrm{N}=1, \\
\mathrm{M}=1\end{array}$ & $\begin{array}{c}\mathrm{N}=1, \\
\mathrm{M}=2\end{array}$ & $\begin{array}{c}\mathrm{N}=2, \\
\mathrm{M}=1\end{array}$ & $\begin{array}{c}\mathrm{N}=2, \\
\mathrm{M}=2\end{array}$ \\
\hline & 2 & 792 & 1161 & 1161 & 1585 \\
\hline
\end{tabular}

\begin{tabular}{|c|c|c|c|c|}
\hline \multicolumn{5}{|c|}{$\begin{array}{c}\text { Table 2: Comparison of Different Antenna } \\
\text { Systems }\end{array}$} \\
\hline Туре & $\begin{array}{c}\text { Tx } \\
\text { Antenn } \\
\text { as }\end{array}$ & $\begin{array}{c}\text { Rx } \\
\text { Antenn } \\
\text { as }\end{array}$ & $\begin{array}{c}\text { Data } \\
\text { Rate } \\
\text { S }\end{array}$ & $\begin{array}{c}\text { Covera } \\
\text { ge }\end{array}$ \\
\hline SISO & Single & Single & Less & Less \\
\hline $\begin{array}{c}\text { MIM } \\
\mathrm{O}\end{array}$ & $\begin{array}{l}\text { Multipl } \\
\mathrm{e}\end{array}$ & $\begin{array}{c}\text { Multipl } \\
\mathrm{e}\end{array}$ & $\begin{array}{c}\text { Great } \\
\text { er }\end{array}$ & Greater \\
\hline
\end{tabular}

\begin{tabular}{|c|c|c|c|c|c|}
\hline 2 & 4 & 1161 & 1585 & 1585 & 2043 \\
\hline 3 & 6 & 1403 & 1850 & 1850 & 2321 \\
\hline 4 & 8 & 1585 & 2043 & 2043 & 2522 \\
\hline
\end{tabular}

There exist a huge number of purposes behind utilizing various radio wire frameworks. There are three essential connection execution parameters that totally portray the quality and value of any remote connection: speed, range (or scope), and unwavering quality (security). The mix of multi receiving wire framework with multicarrier framework gives a superb execution. This area gives a concise review of various systems without guaranteeing to be complete. Basically, two unique classes can be recognized. The main goal is to enhance the connection dependability, that is, the ergodic error probability or the outage probabilities are diminished. This can be refined by improving the momentary signal to noise (SNR) (beam forming) or by diminishing the varieties of the SNR (diversity).

\section{CONCLUSION}

This paper gives the significant highlights innovations and execution of MIMO connects and in addition SISO and MIMO limit correlation for cutting edge remote system frameworks. High information rates and execution of the framework is accomplished by appropriate framework outline of MIMO framework. It is cleared that the accomplishment of MIMO framework joining into business models, for example, 3G, 4G, WiMAX, WLAN, LTE and so forth. Along these lines, at high SNR, the limit increments directly with the quantity of receiving wires at both transmitter and beneficiary side of the MIMO framework. We have examined the conduct of MIMO frameworks that select a subset of accessible radio wires at one connection end. Important applications for such frameworks are cell frameworks with MIMO capacity. The need of choosing reception apparatuses at one connection end (rather than utilizing every one of them) stems either from manysided quality or cost considerations. 


\section{Reference}

1. C. E. Shannon, "Communication theory of secrecy systems," Bell Syst. Tech. J., vol. 28, pp. 656-715, 1949.

2. A. D. Wyner, "The wire-tap channel," Bell Syst. Tech. J., vol. 54 , no. 8 , pp. $1355-$ 1387, 1975.

3. I. Csiszar and J. Korner, "Broadcast channels with confidential messages," IEEE Trans. Inform. Theory, vol. 24, no. 3, pp. 339-348, May 1978.

4. R. Negi and S. Goel, "Secret communication using artificial noise," in Proc. VTC Fall, Sept. 2005, pp. 19061910.

5. S. Goel and R. Negi, "Secret communication in presence of colluding eavesdroppers," in Proc. MILCOM, Nov. 2005, pp. 1501-1506.

6. S. Goel and R. Negi, "Guaranteeing secrecy using artificial noise," IEEE Trans. Wireless Commun. vol. 7, no. 6, June 2008.

7. A. L. Swindlehurst, "Fixed SINR solutions for the MIMO wiretap channel," in Proc. IEEE ICASSP, Taiwan, Apr. 2009, pp. 2437-2440.

8. K. Sengar, N. Rani, "Study and Capacity Evaluation of SISO, MISO and MIMO RF Wireless Communication Systems," IJETT, Vol. 9, No. 9, Mar-2014

9. G. Saini, S. Meghwal, "Capacity Improvement in Multi-User MIMO System using Dirty Paper Coding," IJRITCC, Vol.2, Issue-2, Feb. 2014.

10. A. F. Sengar, N. Rani, "Capacity of MIMO Systems with Antenna Selection," IEEE Transaction, Vol. 4, No. 4, July 2005.

11. A. Goldsmith, "Capacity Limits of MIMO Channels," IEEE Communication, Vol. 21, Issue-5, June 2003.

12. S. Kumar and Deepak Kedia," Study and Performance Analysis of a General MIMOOFDM System for Next Generation Communication Systems," International Journal of Electronics Communication and Computer Technology (IJECCT), Vol. 3, Issue 5, pp. 460-463, Sept. 2013. 